\title{
Ring-down absorption spectroscopy for analytical microdevices
}

\author{
Hans-Peter Loock
}

\begin{abstract}
Ring-down absorption spectroscopy is an emerging "label-free" detection method for analytical microdevices, such as micrototal analysis systems ( $\mu$-TAS). Developed from the related gas-phase cavity ring-down absorption spectroscopy, fiber-optic-based ring-down techniques for liquid samples offer low detection limits, high sensitivity and fast response.
\end{abstract}

(c) 2006 Elsevier Ltd. All rights reserved.

Keywords: Absorption detection; Capillary electrophoresis (CE); Cavity ring-down spectroscopy (CRDS); Fiber-loop ring-down spectroscopy (FLRDS); Microfluidic device; Phase-shift fiber-loop ring-down spectroscopy (PS-FLRDS)

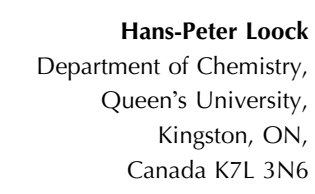

E-mail:

hploock@chem.queensu.ca

\section{Introduction}

In recent years, microfluidic separation techniques have changed the face of analytical chemistry. For example, capillary electrophoresis (CE) has greatly enhanced the sequencing of the human genome [1] and microfluidic chips have permitted not only chemical separation of nanolitersized, complex mixtures [2] but also highthroughput chemical reactions [3].

The main advantages of microfluidic devices, the small sample volume and high separation speed, pose a considerable challenge for detection systems. Currently, fluorescence detection and UV-Vis absorption are the most frequently used methods for CE, with mass spectrometry (MS) forming a valuable alternative for very complex mixtures [4]. Many other detection methods, such as electrochemical techniques, Raman spectroscopy and nuclear magnetic resonance (NMR) spectroscopy, also exist and were reviewed in 2004 by Mogensen et al. [5]

Fluorescence detection is, of course, extremely sensitive - allowing single molecule detection in some circumstances [6,7] - but has the disadvantage that many analytes do not naturally fluoresce, thus requiring the introduction of a labeling step. Since labeling requires sample manipulation, which may introduce errors in the measurements, there have been numerous attempts to find alternatives to fluorescence detection.

Mass spectrometry is a powerful, albeit expensive, method for identifying components in complex mixtures, especially proteins. The interface between microfluidic chips and conventional mass spectrometers previously reduced the time response of the detection, but recent improvements permit the identification of analytes with a separation power that is close to the limiting value for the microfluidic device itself [4]. Recently, miniature mass spectrometers have been developed and successfully tested [5].

Other detection methods, such as electrochemical detection, chemiluminescence, as well as a variety of optical techniques, based, for example, on the measurement of refractive index, scattering, and surface plasmons, have also been investigated and were the subject of recent reviews $[5,8]$.

For many chromatographic applications, absorption measurements appear to be the most straightforward detection method. For label-free detection, UV-Vis absorption has been used most widely due to its universality, sensitivity and fair limit of detection (LOD) $\left(10^{-5}\right.$ to $\left.10^{-6} \mathrm{M}\right)[9]$. Many analytes absorb light in the near-UV region and, by selecting a spectral window in which the solvent is transparent but the majority of analytes are not, one could envisage a universal, label-free detection scheme. However, absorption measurements in microfluidic devices present a considerable challenge, since the absorption paths correspond to the width of the separation channels, the analyte is dilute, and the time response required is of the order of milliseconds, with no possibility of averaging multiple separation events. 


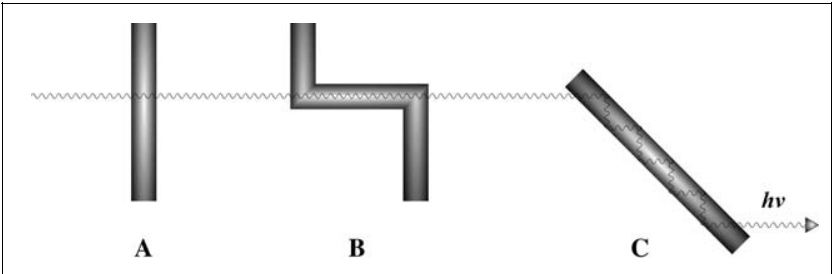

Figure 1. Enhancement of absorption path compared to single pass absorption (A), Z-cell (B), and multipass reflection cell (C).

Consider that capillaries used for CE typically have an inner diameter of about $100 \mu \mathrm{m}$, and microfluidic channels have typically dimensions of $30 \mu \mathrm{m} \times 50 \mu \mathrm{m}$. These short absorption path-lengths result in poor sensitivity, especially for analytes with low molar extinction coefficients $[10,11]$.

In addition, low-loss coupling of absorption spectrometers to microfluidic devices is not easy. For example, a typical single-pass bench-top spectrometer using an incoherent light source has a minimum detectable absorption loss, of $\alpha_{\min }=(e C)_{\min }=10^{-3}-10^{-4} \mathrm{~cm}^{-1}$. This is orders of magnitude better than what can reasonably be expected from an online absorption detector in a microfluidic device. However, chips that make use of integrated glass or polymeric waveguides are likely to provide improved coupling for absorption measurements in the near future. Currently the loss of these waveguides is with $1 \mathrm{~dB} / \mathrm{cm}$ quite high, ${ }^{1}$ and a comparably large fraction of the optical loss occurs in the waveguide, thereby masking the smaller optical loss caused by analyte absorption.

A conceptually simple way to increase the absorption path is the introduction of 'Z-cells' [12,13], 'U-cells' [14] or bubble cells $[15,16]$ into the microchannel (Fig. 1) While these cells are straightforward to implement in a microfluidic device - although maybe less straightforward to interface to a spectrometer - the enhanced absorption path comes at the cost of reduced temporal resolution and therefore loss in separating power. Alternatively, multi-reflection nanoliter-scale cells require exact, robust alignment of a highly-collimated light source. With any change in incidence angle or even refractive index of the solution, the absorption path changes too, and the measured absorption loss fluctuates [13,17].

Finally, all conventional "short-path" absorption techniques suffer from problems associated with measuring small intensity changes in the presence of a relatively large intensity background and in the presence of background absorption losses due to interfacing with the light source and detector. This problem is compounded by the fact that lasers, as intense light sources, may

${ }^{1}$ Related to the transmission by $\mathrm{T}=10^{-\mathrm{dB} / 10}$. exhibit intensity fluctuations with time and laser wavelength. Absorption sensor arrays that simultaneously probe different regions of the flow channel (e.g., using array detectors combined with spatially extended light sources [18]) differentiate between the signals and thereby circumvent some of the stability problems. Nevertheless, the lack of sensitivity and robustness are still considered fundamental limitations of absorption detection techniques in microfluidic applications.

\section{Cavity ring-down spectroscopy}

A similar problem of measuring a small optical loss has been elegantly addressed by cavity ring-down spectroscopy (CRDS) [19-23], a multipass gas-phase absorption technique that is largely insensitive to light-intensity fluctuations, since the optical loss (absorption) is inferred from the rate of the intensity change rather than absolute intensity. Typically, a CRDS system comprises a pulsed laser as a light source, a gas-containing cavity with two highly reflective mirrors and a sensitive light detector (e.g., a photo multiplier tube). The laser light pulse is directed at the back of one of these mirrors and a small fraction of the light pulse enters the cavity, where it is repeatedly reflected between the two mirrors. A sensitive photon detector is placed behind the second mirror and monitors the light that leaks out of the cavity as a function of time. After the laser pulse has been introduced into the cavity, the rate of intensity loss inside the cavity depends on the quality of the mirrors and the presence of optical absorbers in the cavity. From an exponential fit to the envelope of the intensity of the light emitted from the cavity, one can then determine the exponential decay time. This ring-down time (RDT), only depends on the optical loss processes inside the cavity, and, ideally, not the intensity of the incoming laser light pulse. Most importantly, in a cavity filled with an absorbing medium, the sensitivity and the LOD of the absorption measurement are enhanced by the increased effective absorption path length. Conveniently, the sensitivity of the RDT measurement is greatest when the optical absorption approaches zero [24]. For very highfinesse cavities, effective absorption paths of several tens of kilometers have been achieved with bench-top instruments [21]. Several companies now offer cavity ring-down spectrometers as tools for atmospheric spectroscopy, air-quality measurements and other applications.

The RDT of such a cavity is given by [19]:

$\tau=\frac{L}{c\left(-\ln R+\sum_{i} \varepsilon_{i} C_{i} d\right)}$

where $c=c_{0} / n$ is the speed of light, $L$ is the length of the cavity, $d$ is the length of the sample path, which is 
identical to $L$ if the entire cavity is filled with sample, and $R$ is the reflectivity of the mirrors. Note that $(1-R) \approx-\ln R$ for $R \approx 1$. Reflectivities of better than $R=0.9999$ can be achieved by commercial mirrors and some "supermirrors" have an optical loss in the singledigit ppm range. The tuning range of these mirrors is typically of the order of 3-9\% of the center wavelength. Assuming a cavity length of $1 \mathrm{~m}$, RDTs of empty cavities of tens of microseconds are routinely achieved corresponding to several thousand passes through the gas sample. Minimal detectable absorption losses of $(e C)_{\min }=\alpha_{\min }<10^{-7} \mathrm{~cm}^{-1}$ are possible in this pulsed CRDS operation [25].

Many variants of CRDS have been developed over the past 15 years or so. For example, by exciting a singlecavity mode, one observes a much longer RDT, but at the price of having to change the length of the cavity synchronized to the laser wavelength. More commonly, many researchers excite the cavity non-resonantly into a pseudo-continuum of modes [25]. Also, as an alternative to laser-pulse excitation, continuous-wave $(\mathrm{cw})$ excitation may lead to cavity build-up, if the cavity happens to drift into resonance with a single mode, whereupon the laser is switched off and a ring-down is observed. The photon lifetime in the cavity can then be determined from either the build-up or ring-down of the cavity [26]. A related method - phase-shift CRDS (PSCRDS) - infers RDTs from the difference in phase of intensity-modulated light entering and exiting the cavity. Details of this technique are relevant to one of the fiber-optic techniques that we developed and will be discussed below. The reader is referred to a 2005 historical overview of cavity ring-down methods for a detailed comparison of these and related techniques [27].

How can CRDS be adapted for condensed phase absorption measurements? In a most straightforward application, Zare and co-workers [29] filled their entire $20-\mathrm{cm}$ cavity with a solution of organic or inorganic absorbers in a variety of organic solvents, and were able to probe, for example, $1-10 \mathrm{pM}$ solutions of these strong absorbers, corresponding to $\alpha_{\min }=10^{-6} \mathrm{~cm}^{-1}$. These experiments showed not only a certain disrespect for the high-reflectivity mirrors - every CRDS spectroscopist's obsession - but also that CRDS of solutions is feasible in the standard CRDS configuration (Fig. 2). ${ }^{2}$

In 2005, Bahnev et al. presented a cavity that was shrunk to dimensions of a capillary cross section and showed that CRDS detection of analytes separated by HPLC is feasible [28]. The RDTs scale linearly with the cavity length and were consequently of the order of

\footnotetext{
${ }^{2} \mathrm{~A}$ major practical limitation of CRDS is that mirrors are exquisitely sensitive to optical imperfections and deposits.
}

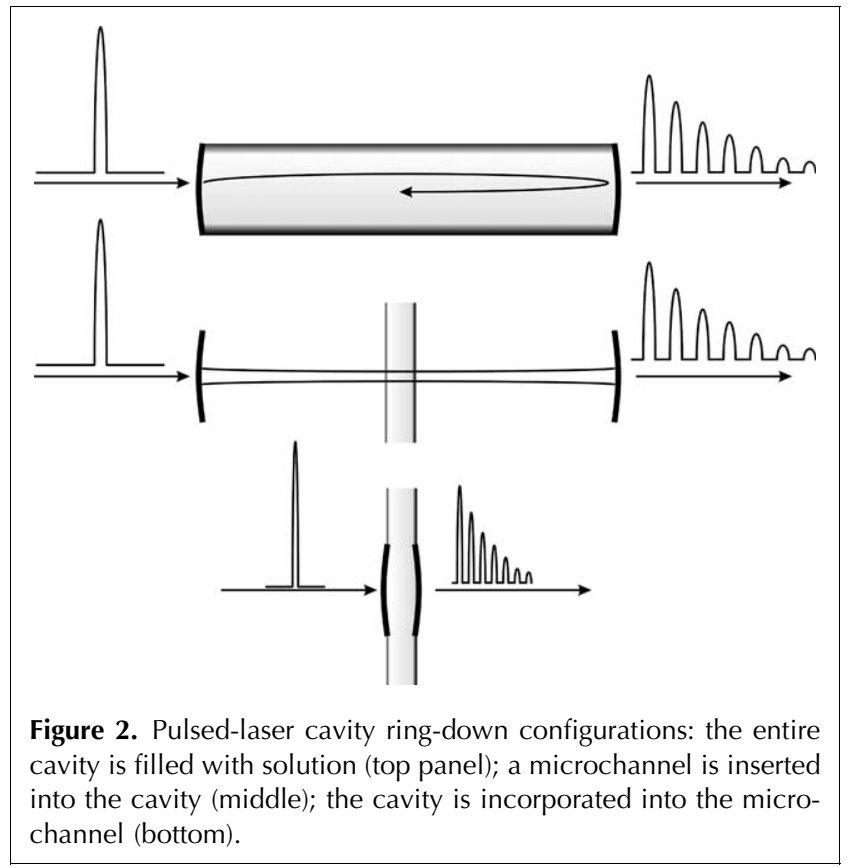

nanoseconds, thus requiring ultrafast laser and detection systems.

$\mathrm{Xu}$ et al. realized CRDS liquid-phase measurement by inserting two standard UV-Vis cuvettes into a 1-m cavity at Brewster's angle [29]. It was noted that the refractive index of the liquid sample measured must be close to that of the cuvette, and aqueous samples therefore produce additional optical losses. Another successful application of CRDS as a chromatographic detector was developed, again by the Zare group [30]. In their application, a special Brewster-angled flow cell was placed in a 1-m cavity and absorption measurements from samples separated by HPLC were obtained.

Other approaches to condensed-phase CRDS measurements include deposition of a thin film onto the mirror surfaces [31] and situating a thin film in a large cavity on a transparent support that is either perpendicular to the cavity axis [32,33] or at Brewster's angle [34]. It should also be mentioned that CRDS measurements were carried out using a monolithic crystal as a cavity to probe the absorption by the evanescent wave [35-38]. Extraordinary LODs were achieved in this way, allowing the detection of $0.006 \%$ of a monolayer of $\mathrm{I}_{2}$ adsorbed on the cavity monolith [37].

\section{Ring-down spectroscopy using waveguides}

Of special interest in this article are ring-down measurements using optical fiber "cavities". In the following paragraphs, we group those "wave-guided cavities" into linear fiber cavities and fiber loops. Linear fiber strands can become cavities, for example by coating both ends with reflective materials in an approach developed in Sigrist's group. The reflectivity of those custom mirrors 
was a respectable $R=0.995$, and evanescent wave absorption [39] as well as the diffusion kinetics of $\mathrm{H}_{2}$ into the fiber were observed [40]. At about the same time, Gupta et al. presented a cavity that made use of two identical highly reflective fiber Bragg gratings as "mirrors", also sensing the evanescent wave [41]. Since the fibers used by both groups had a small core diameter and therefore only supported a single mode, operating the cavity in a mode-locked fashion was comparably easy.

An alternative fiber-optic ring-down technique makes use of loops of optical waveguides. In 2001, Stewart et al. coupled light from a pulsed laser into a $\sim 60 \mathrm{~m}$ loop of optical fiber and obtained the photon lifetime from RDT measurements [42]. In their implementation, a gasabsorption cell was inserted into the loop to allow for gas sensing. Since the insertion loss of the micro-optic gas cell was rather large, it became necessary to include a fiber amplifier into the loop, which operated just below the threshold for net amplification of light in the loop. Very long RDTs were observable, but the accuracy of the measurement then relied on the stability of the amplifier. In an extension of this work, the same group has recently achieved RDTs of milliseconds for these amplified fiber-loop systems [43].

Our group has developed a similar approach based on passive (non-amplified) ring-down measurements in optical waveguide loops. In our experiments, a small gap was left between the two ends of the fiber-optic cable and a sample was introduced into this gap. Absorption measurements of picoliter-sized samples of a dilute dye solution were possible and yielded an LOD below 100 attomoles for a strong near-IR absorber [44].

A similar approach by the Lehmann group also made use of an optical-fiber loop, but with the difference that the absorption of the evanescent wave outside the fiber was detected. Here, a small segment of the fiber loop was tapered such that the evanescent wave of the core mode was exposed [45]. Their method permitted the detection of a single-cell adsorption event.

Passive fiber-loop ring-down spectroscopy (FLRDS) was also used by Wang and Scherrer as a means to determine pressure and strain [46]. As described earlier by Sigrist [39] and us, mechanical deformation of the fiber dramatically increases the optical loss of the fiber cavity and hence reduces the RDT. Macrobending losses show a steep onset at a critical radius that for many fibers is in the vicinity of $2-3 \mathrm{~cm}$. Controlled physical deformation of the waveguide can be quantified by ringdown spectroscopy and hence permits the use of optical fibers as mechanical transducers. Many of the techniques described above were the subject of an excellent 2005 review [47].

In the following, we will focus on FLRDS, in which a fiber-loop is used instead of the mirrors to construct a ring-down "cavity". A gap left between the two fiber ends allows for introduction of small volumes of liquid, making the detector suitable for use with analytical devices such as capillary electrophoresis (CE) and micro-total analysis systems ( $\mu$-TAS). Nanosecond laser pulses are injected into the single-mode or multi-mode fiber, and can be observed for typically 100-200 round trips. With each round trip, the laser pulse passes through the sample. The fractional loss in laser intensity is detected by a fast photodetector. The technique is not necessarily superior to the other techniques described before, but has the advantage of being very inexpensive, robust, inherently size-matched to microfluidic channels, and simple to implement.

By analogy to Equation (1), the RDT of the loop can be described by a single exponential decay:

$\tau=\frac{n L}{c_{0}\left(-\ln (T)+\alpha L+\sum_{i} \varepsilon_{i} C_{i} d\right)}$

where $e_{i}$ is the extinction coefficient of an absorbing species $i, C_{i}$ is its concentration and $d$ is the width of the absorption cell formed by the two fiber ends. The absorption losses of the fiber material are characterized by the length of the fiber loop, $L$, and the absorption coefficient of the fiber material, $\alpha$. Finally, the speed of light in the fiber, $c_{0} / n$, is determined by the refractive index of the fiber core ( $n \approx 1.45$ for silica), and $T$ is the transmission of the fiber-fiber joint in the absence of any absorber.

The intensity of the round trips seen in Fig. 3 follows a bi-exponential decay, which arises from the fact that light is guided both by the core and the cladding of the fiber, and that core and cladding modes decay with different RDTs (here, $900 \mathrm{~ns}$ and $235 \mathrm{~ns}$, respectively). The

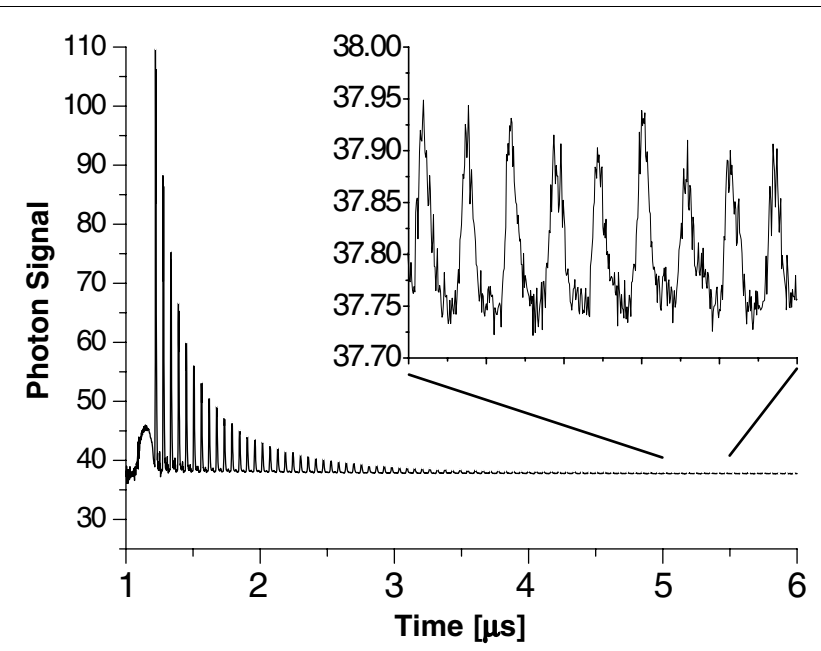

Figure 3. Average ring-down waveform obtained from a $12-\mathrm{m}$ fiber-loop. The round trips are separated by about 60 ns and form a biexponential decay with RDTs of 235 ns and 900 ns, corresponding to the decay of cladding and core modes. Waveforms from about 5000 laser shots were averaged. (Reproduced with permission from the Journal of Chemical Physics). 
presence of an analyte introduces additional optical loss and hence shorter RDTs. The sample volume is only about $7 \mathrm{pL}$ and the absorption path is $d=3.8 \mu \mathrm{m}$. A detailed study gave the LOD at $\alpha_{\min }=100 \mathrm{~cm}^{-1}$ and an enhancement of the absorption path by about two orders of magnitude.

In addition to its potential as an absorption spectroscopic technique, FLRDS may be used to characterize the low insertion loss of fiber-optic components, such as filters, couplers, splices and fiber gratings.

The application of FLRDS to on-line detection of separation events in microfluidic devices faces a number of obstacles. First, the time-response of the detection has to be reduced from about $30 \mathrm{~s}$ per measurement for pulsed operation to less than $1 \mathrm{~s}$ to match the separation speeds of microfluidic devices. Second, the system has to be made compact and inexpensive, and, finally, the detection wavelength has to be moved into a region in which many analytes, but not many solvents, absorb. As shown below these problems can be addressed by changing the mode of detection and the laser light source.

In gas-phase CRDS, $c w$ laser-light sources are increasingly used, mainly because of their lower cost and narrower line width. Here, the build-up and/or ringdown of the cavity is monitored for events in which the excitation laser accidentally matches a cavity mode. Obviously, this mode of operation is not suitable for FLRDS experiments in which multimode fibers are used. However, a related technique, introduced by Meijer and co-workers, makes use of an intensity modulated light source to obtain high duty cycles and therefore a very fast time-response [48]. This technique was adapted by our group and it was shown that "phase-shift FLRDS" (PS-FLRDS) gives not only a time response of milliseconds but also a lower LOD in the detection of small amounts of liquid samples [49]. Similar to the equivalent gas-phase technique [48], in PS-FLRDS, an intensitymodulated $c w$ laser with angular modulation frequency, $\Omega$, illuminates the fiber loop. The phase difference, $\Delta \Phi$, between the light entering the loop and exiting the loop is then related to the RDT, $\tau$, by $\tan (\Delta \Phi)=-\tau \Omega$.

Phase-angle measurements have considerable advantages over pulsed RDT measurements: the method has a time response that can be a high as a few $\mathrm{kHz}$, the device can be made very compact, the data requires no fitting, and, since the duty cycle is close to $100 \%$, weak light sources $(P<3 \mathrm{~mW})$ are sufficient. In the present study, the data-acquisition time was set to $300 \mathrm{~ms}$, as a compromise between high number of averaged cycles (high $\mathrm{S} / \mathrm{N}$ ratio) and a fast acquisition rate, which is comparable to the response of a conventional UV-Vis detector.

\section{FLRDS detection in analytical microdevices}

In the following, we describe three microanalytical devices in which PS-FLRDS has been used as a fast absorption detector.

\subsection{Fiber loop}

Typically a 10-80-m long multimode optical fiber was used to make a fiber loop (Fig. 4). Since the attenuation of our fiber is less than $2 \mathrm{~dB} / \mathrm{km}$ at around $800 \mathrm{~nm}$,

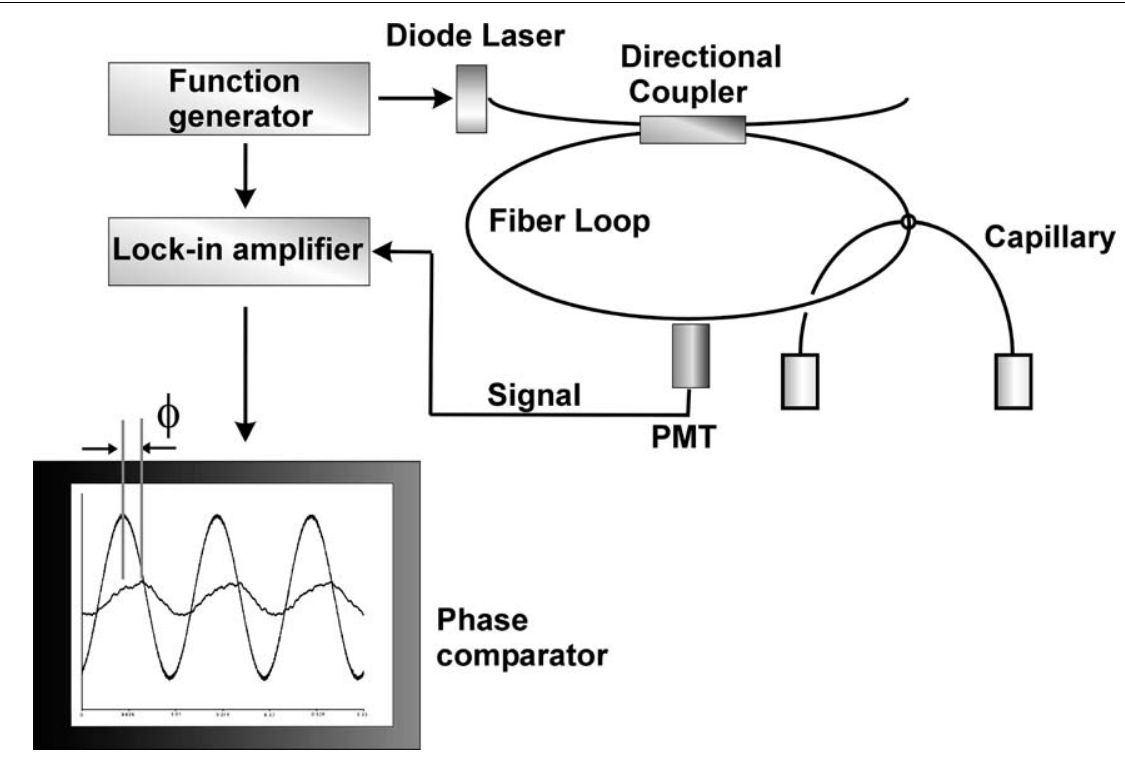

Figure 4. Phase-shift fiber-loop ring-down spectrometer interfaced with a capillary flow system. The phase angle between the incoming and emitted light from the fiber loop is a simple measure for the optical loss and is obtained using either a fast lock-in amplifier or another phase comparator. 
there is no disadvantage in using such long "cavities". For commercial silica fibers the attenuation of the waveguide material itself is very small in the 800-1550 $\mathrm{nm}$ region and typically less than $2 \mathrm{~dB} / \mathrm{km}$ (or $0.5 \%$ for a 10-m fiber loop). However, even specialty UV fibers have a much larger loss in the UV-Vis region - typically about $10 \mathrm{~dB} / \mathrm{km}$ at $500 \mathrm{~nm}(2.2 \%$ loss for $10 \mathrm{~m})$ and $100 \mathrm{~dB} /$ $\mathrm{km}$ at $300 \mathrm{~nm}(20 \%$ loss for $10 \mathrm{~m})$, whereas specialty mid-IR fibers with similar losses of presently $100 \mathrm{~dB} / \mathrm{km}$ may make measurements at around $3 \mu \mathrm{m}$ possible in the future.

The 810-nm laser diode was current modulated at $100 \mathrm{kHz}$. To couple the laser light into and out of the optical fiber loop, different approaches can be used. One can simply and inefficiently focus the laser output onto the end of an optical delivery fiber and use this fiber to introduce the light into the loop by illuminating a small section of the fiber loop. Index-matching gel can be used to help couple the laser light into the fiber loop. A photomultiplier tube is placed at a different location in the loop and monitors the emission from the loop by detection of photons scattered from the fiber. Alternatively, commercial 2:2 fiber-fiber couplers permit a more efficient, stable means to deliver light into the loop, and a 2:1 fiber tap can be used to leak light into the detector (see Fig. 4). However, many couplers have insertion losses of a few percent that ultimately limit the background transmission of the loop.

The signal from the photodetector is fed into either a fast lock-in amplifier or a home-built phase comparator chip, and the phase is referenced to the driving current of the laser diode. To reduce electromagnetic interference, all cables are shielded.

The phase-shift, $\Phi$, and RDT, $\tau$, are related by [48]:

$\Phi-\Phi_{0}=-\arctan (\Omega \tau)$

where $\Phi_{0}$ is the phase angle in the absence of analyte and $\Omega$ is the modulation frequency of the $c w$ laser. This offset angle, $\Phi_{0}$, reflects the time delays in signal acquisition and processing and, while the value is constant for each configuration, it is difficult to predict. To determine $\Phi_{0}$, Equation (3) can be rewritten [44]:

$\Phi=\Phi_{0}-\arctan \frac{n \Omega L}{c_{0}(A+\varepsilon d C)}$

where $L$ is the length of fiber loop, $n=1.46$ is the refractive index of the core modes, $c_{0}$ is the vacuum velocity of light, $A=-\ln T+\alpha L$ is a constant related to the FLRDS system, $e$ is the molar extinction coefficient of samples with respect to base $e$, and $d$ is the size of the detection gap. The modulation frequency was chosen such that $\Omega \approx(2 \pi \tau)^{-1}$ [48]. Calibration curves obtained by filling three different microdevices with solutions of different concentration are displayed in Fig. 5 and were used to determine $A, d$ and $\Phi_{0}$ by fitting.
Equation (3) illustrates that a linear relationship exists between $1 / \tan \left(\Phi_{0}-\Phi\right)$ and concentration. An electrophoregram/chromatogram is then simply constructed by graphing $1 / \tan \left(\Phi_{0}-\Phi\right)$ as a function of time.

Cyanine dyes ADS832WS and ADS805WS (American Dye Source) were selected as analytes to test the performance of the PS-FLRDS system because their maximum absorption wavelength is close to the laser wavelength $(810 \mathrm{~nm})$. The laser dyes were used without further purification and had an absorption coefficient of $e=\ln (10) 1.6 \times 10^{5} \mathrm{M}^{-1} \mathrm{~cm}^{-1}$ and $e=\ln (10) 1.9 \times 10^{5}$ $\mathrm{M}^{-1} \mathrm{~cm}^{-1}$, respectively.

It may not be immediately apparent whether reliable phase-shift measurements can be made, if the optical decay is not a single exponential. In loops made of multimode fibers, multi-exponential decays are a concern, since comparable intensity is carried in the cladding and core of the fiber, and the intensity of the cladding and core modes decay at different rates. Nevertheless, it was found that bi-exponential decays in fiber cavities using phase-angle measurements can be fully characterized by measuring phase angles as a function of modulation frequency. It also became apparent that there is an advantage to using modula-

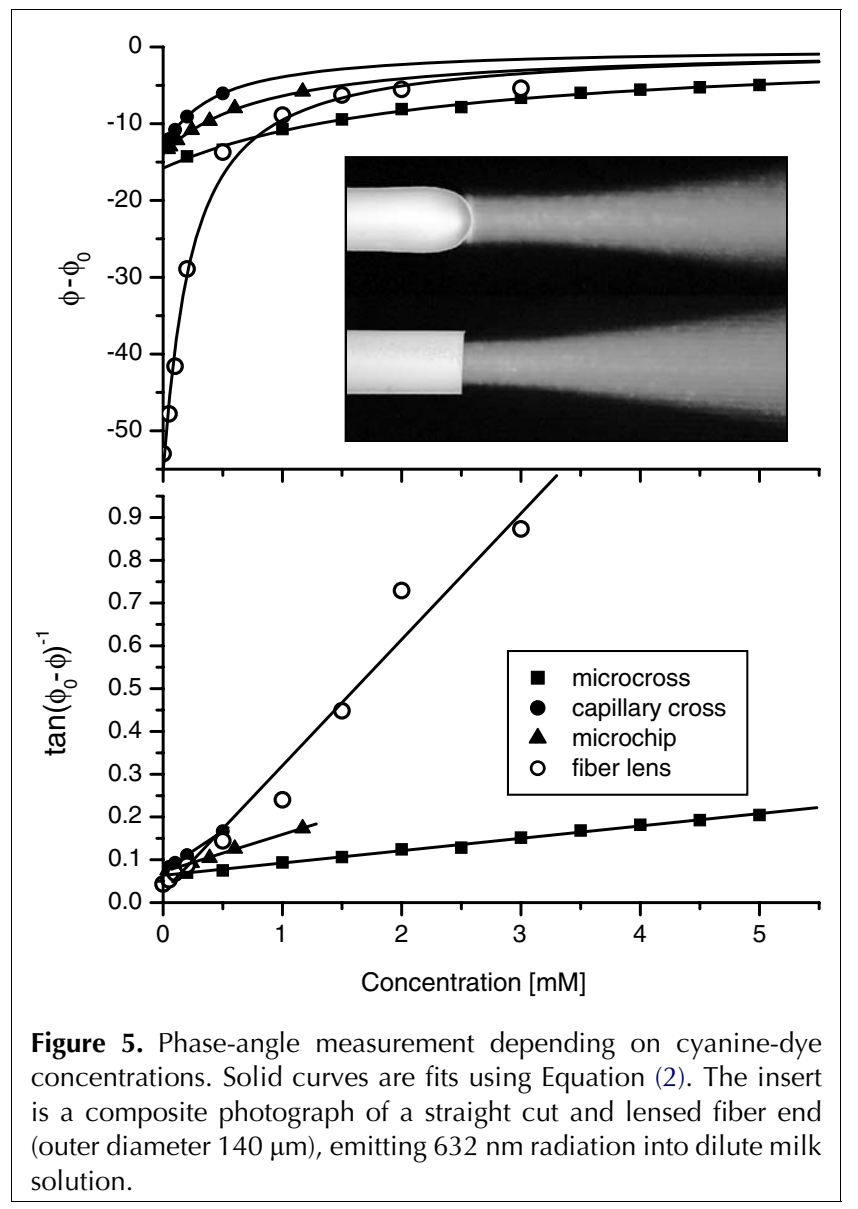


tion frequencies that are considerably lower than $\Omega \approx(2 \pi \tau)^{-1}[44]$.

\subsection{Capillary flow using a microcross interface}

The simplest interface between capillary and fiber-loop, shown in Fig. 6, made use of a commercially available microcross into which the fiber ends and the two capillary sections were inserted at right angles to each other. The two fiber ends were therefore directly in the flow path. The fiber ends were aligned to be within $d=5-50$ $\mu \mathrm{m}$ of each other. The dead volume of such a device is 38 $\mathrm{nL}$, which is high but acceptable for many applications.

A series of $2 \mu \mathrm{L}$ solutions with different concentrations of ADS832WS dye $(0.2-5.0 \mathrm{mM})$ was injected into the microcross detection gap and a typical flow trace is shown in Fig. 6. Fair repeatability of peak areas was

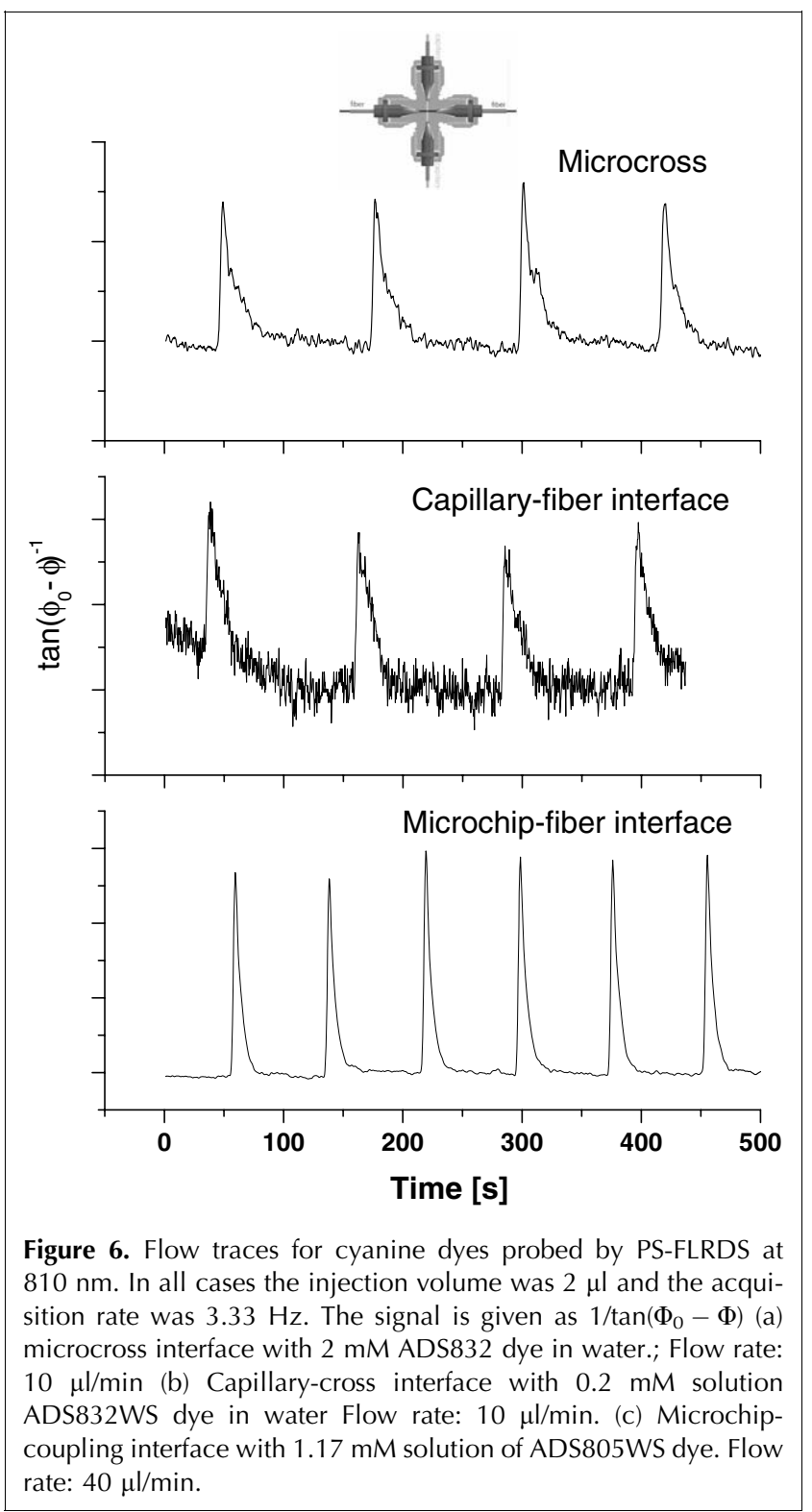

obtained with the relative standard deviation of $6 \%$. The LOD of the microcross PS-FLRDS system can be calculated as $200 \mu \mathrm{M}\left(\alpha_{\min }<50 \mathrm{~cm}^{-1}\right)$, which is not adequate for most electrophoretic measurements. By comparison with experiments using translation stages, it can be concluded that the poor performance of the commercial microcross was due to the difficulty in aligning the fiber ends accurately. Alignment of the center axis to within better than $5 \mu \mathrm{m}$ is essential for good performance, but difficult using a microcross.

\subsection{Capillary-flow using inserted-fiber interface}

To permit a more precise alignment of fiber and capillary, an interface was made by drilling a $150-\mu \mathrm{m}$ channel through the center of the fused silica capillary (I.D. $100 \mu \mathrm{m} /$ O.D. $360 \mu \mathrm{m}$ ). First, the capillary was embedded in PMMA polymer by drilling a $360-\mu \mathrm{m}-$ diameter hole into a block of PMMA and inserting the capillary into the hole. The capillary was then fixed using epoxy glue. Then, with the aid of a microscope, a $150-\mu \mathrm{m}$ hole was drilled perpendicular to the long axis of the capillary through the PMMA and through the capillary (Fig. 7). Here, a commercial 150- $\mu$ m drill bit (Drill Technology, Ada, Michigan, USA) was used. The two ends of the fiber loop were then inserted into the precision-drilled hole and aligned to let the fiber ends meet with the inner capillary wall $(\sim 60 \mu \mathrm{m})$. Finally, the capillary-fiber interface was sealed by epoxy glue.

This interface was tested by using a solution of ADS832WS dye in water. A typical flow trace and peakarea calibration curve of the capillary-cross PS-FLRDS system are shown in Fig. 6. Again, good repeatability was obtained with the average relative standard devia-

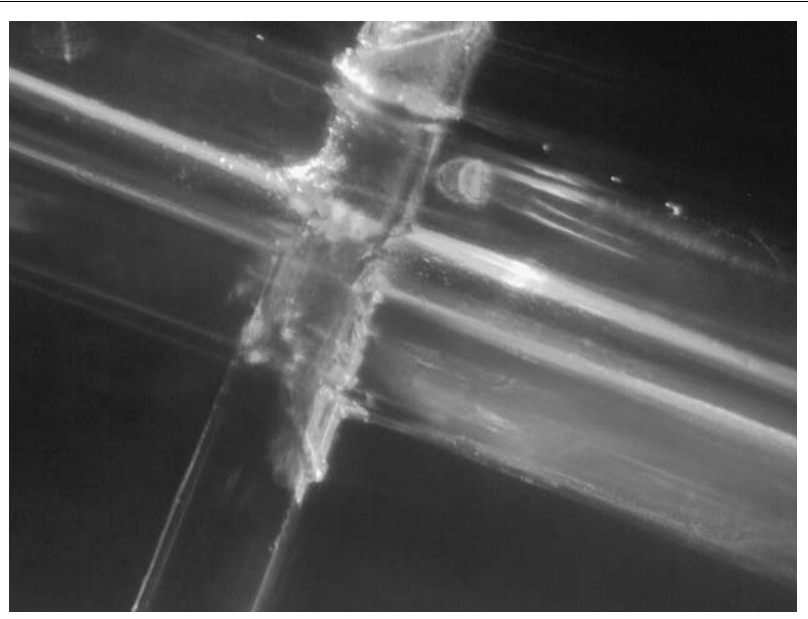

Figure 7. Interface of a 360/100- $\mu \mathrm{m}$ capillary with a $140 / 100-\mu \mathrm{m}$ fiber loop. The capillary was embedded into a block of PMMA polymer and $150-\mu \mathrm{m}$ hole was drilled through the polymer block and capillary. The two ends of a fiber were then inserted and sealed with epoxy glue. 
tion of the peak areas of $2 \%$. In the concentration range of $0.05-0.5 \mathrm{mM}$, the peak areas show a linear response to the concentrations of ADS832WS $\left(R^{2}=0.999\right)$. Furthermore, The LOD was improved over the microcross arrangement to $50 \mu \mathrm{M}\left(\alpha_{\min }=10 \mathrm{~cm}^{-1}\right)$.

\subsection{Microchip-flow using inserted-fiber interface}

The fiber loop was also coupled to a commercial microfluidic chip and aligned normal to the chip surface. Downstream of a feed capillary, a 150- $\mu$ m hole was drilled through the microchannel $(50 \mu \mathrm{m}$ wide and 20 $\mu \mathrm{m}$ deep) and out through the bottom face of the chip. To protect the microchannel from clogging while drilling, high pressure was applied to the water in the microchannel using a syringe pump. The two ends of the fiber loop were inserted into the fiber channel. The distance between the fiber ends was adjusted to about 10 $\mu \mathrm{m}$. Finally, the microchip-fiber interface was sealed by epoxy glue (Fig. 8).

To test the performance of the microchip-coupling device, ADS805WS dye was used. A typical flow trace
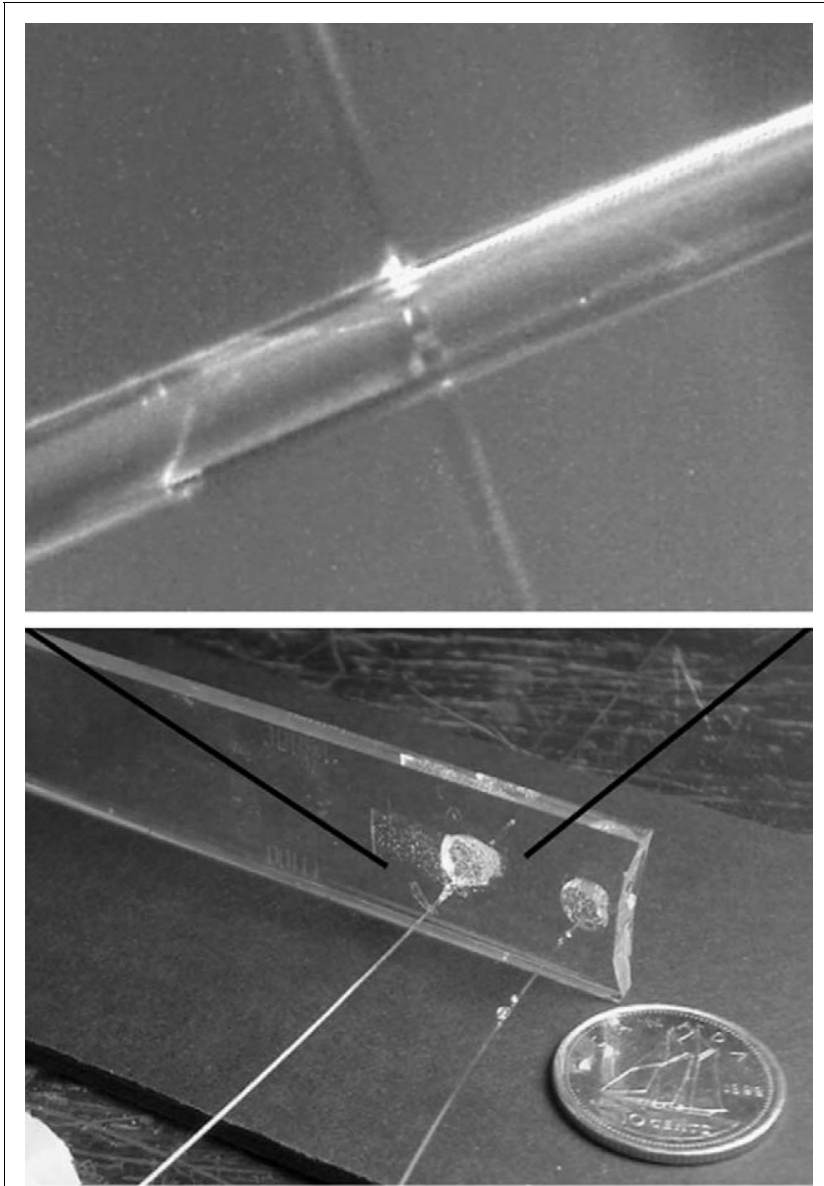

Figure 8. The microchip/fiber-loop interface: the top panel shows the drilled hole with the inserted fiber (horizontal) and the microchannel (vertical). The bottom panel shows the finished chip interfaced to the fiber loop and the feed capillary. and the calibration curve are shown in Fig. 6. Very good repeatability was obtained with the relative standard deviations of peak areas and peak heights of $0.93 \%$ and $0.13 \%$, respectively. A good response of peak areas to concentrations of ADS805WS was obtained in the range $58 \mu \mathrm{M}-1.17 \mathrm{mM}\left(R^{2}=0.9918\right)$. Based on the signal-tonoise ratio of 3, the LOD can be calculated to be $\sim 30 \mu \mathrm{M}$ $\left(\alpha_{\min }<10 \mathrm{~cm}^{-1}\right)$. From the size of the detection gap of $14 \mu \mathrm{m}$, which was obtained from the fit in Fig. 6 and the channel cross section of $50 \mu \mathrm{m}$, a sample volume of 700 $\mathrm{pL}$ could be estimated. Given an LOD of $30 \mu \mathrm{M}$ solution, we can therefore detect 21 femtomole of ADS805WS using the microchip-coupling device - an improvement by one order of magnitude over the capillary/fiber interface described above. The results demonstrate the sensitivity improvement that is possible by decreasing the distance between fiber ends, a parameter that is related to the fiber-fiber coupling efficiency.

\subsection{Lensed fiber ends}

From the parameter $A=-\ln T+\alpha L$, obtained by fitting, it was apparent that the fiber-flow channel interface had a considerable background loss of $(1-T)=0.5-0.6$. Since the absorption of the fiber material is less than $\alpha=0.008$ $\mathrm{m}^{-1}$, the largest contributions are optical coupling losses at the interface with the flow system. In an attempt to reduce these losses, the emitting fiber end was subjected to a brief electrical arc, which caused the fiber end to melt into a convex lens with a radius of $76 \mu \mathrm{m}$.

Calibration curves were obtained using a xyz-translation stage for alignment and were fitted using Equation (4). Here, the straight-end and lensed-end interfaces gave similar values for $\Phi_{0} \approx 25^{\circ}$. The widths of the gaps, $d($ straight $)=86 \mu \mathrm{m}$ and $d($ lens $)=35 \mu \mathrm{m}$, correspond to interaction volumes of 675 and 275 femtoliters, respectively. However, the values for $A=0.58$ and 0.17 , for the straight-cut and lensed fibers, respectively, are dramatically different from each other, indicating that the lensed fiber interface has a much improved coupling efficiency. Assuming that the value for the fiber attenuation, $\alpha_{\text {fiber }}=0.0078 \mathrm{~m}^{-1}(3.4 \mathrm{~dB} / \mathrm{km}$; given by the manufacturer's specifications) is the same for both loops, one can readily determine the coupling efficiency of $T_{\text {(straight) }}=0.60$ and $T_{\text {(lens) }}=0.90$. The RDTs associated with these background losses (i.e. at $C=0$ ) are $\tau=670$ ns and $2.3 \mu \mathrm{s}$, respectively. The larger transmission for the lensed fiber end is largely due to the focusing effect, and not due to the difference in the size of the gap.

From the fitting parameters, we can further estimate the LOD and dynamic range of the concentration measurements. With a measurement accuracy of $\delta \Phi=1^{\circ}$, the LOD for the lensed-fiber setup is $5 \mu \mathrm{M}$ for ADS805 dye (i.e. $\alpha_{\min }=0.8 \mathrm{~cm}^{-1}$ ). At the instrumental limit of $\delta \Phi=0.05^{\circ}$, the LOD corresponds to $240 \mathrm{nM}$ $\left(\alpha_{\min }=0.04 \mathrm{~cm}^{-1}\right)$. This is a considerable improvement over the straight-cut fiber LODs of $10 \mu \mathrm{M}\left(\alpha_{\min }=\right.$ 
$\left.1.6 \mathrm{~cm}^{-1}\right)$ at $\delta \Phi=1^{\circ}$ and $450 \mathrm{nM}\left(\alpha_{\min }=0.08 \mathrm{~cm}^{-1}\right)$ at $\delta \Phi=0.05^{\circ}$. The dynamic range can be calculated using the same measurement accuracy of $\delta \Phi=1^{\circ}$ and is $10 \mathrm{mM} / 5 \mu \mathrm{M}=2000$ for the lensed fiber and $4 \mathrm{mM} /$ $10 \mu \mathrm{M}=500$ for the straight-cut fiber end.

Experiments using lensed fiber ends that are interfaced to flow channels are currently under way. We note that the cyanine dyes used in this work are common noncovalent labels for the analysis of proteins. The PSFLRDS technique could become a sensitive detection method for analyzing proteins and possibly DNA.

\section{Conclusions}

When monitoring separation events in microfluidic devices, one frequently needs to detect small amounts of analyte in picolitre-sized volumes with a time response of milliseconds. We presented an optical detection method that may provide an alternative to fluorescence detection in some cases. This absorption technique is based on the RDT of an optical signal in a cavity or loop made of waveguide material. This cavity contains the flow cell and may be formed, for example, by high-reflectivity mirrors or by an optical waveguide loop.

The optical decay constant of the cavity changes as small liquid samples containing absorbing species are introduced into the flow cell. It is demonstrated that one can obtain the optical decay constant using either a pulsed or an intensity-modulated $c w$ laser beam that is coupled into the cavity.

In the $c w$-CRDS method, the inherent exponential decay of the cavity that contains the microanalytic cell introduces a phase shift of the light emitted from the loop with respect to the pumping beam. By measuring this phase shift, one can readily determine the concentration of the analyte introduced between the two fiber ends. In particular, it is demonstrated that "phase-shift fiber-loop ring-down spectroscopy" (PS-FLRDS) is well suited as an absorption technique for any flow system in which the optical absorption path is limited by the instrument architecture. We briefly outlined three different types of interfaces to capillaries and microfluidic devices and presented a characterization of each interface. Finally, we introduced an improved method of coupling the fiber loop to the capillary, which made use of a lensed fiber. This configuration compared favorably to the previously presented straight-cut fiber ends.

At present, the PS-FLRDS technique is limited to the $800 \mathrm{~nm}$ region of the spectrum. Both UV detection at $\lambda<300 \mathrm{~nm}$ and NIR detection at around $\lambda=1.5-1.7$ $\mu \mathrm{m}$ are suitable for general absorption detection, although the former is limited by the reduced transmission of optical waveguides in the UV region and the latter suffers from the small absorption cross section of vibrational overtone bands.
Nevertheless, we believe that FLRDS in both timeresolved (pulsed-laser) and phase-resolved ( $\mathrm{cw}$ laser) versions may find applications, especially in the detection of non-fluorescent biomolecules that have large UV or NIR absorption cross sections.

\section{Acknowledgement}

Many of the data presented were obtained by Runkai Li and Professor Richard D. Oleschuk with technical assistance from Zhaoguo Tong, Nicholas Trefiak and Daniel Courtney. The FLRDS technique was co-invented with Professor R. Stephen Brown and the author acknowledges numerous discussions with the research groups of Profs. Brown and Oleschuk (Queen's University). Financial support from the Natural Science and Engineering Research Council, Premier's Research Excellence Award, Photonics Research Ontario, Queen's University, and the Canadian Initiative for Photonic Innovations is gratefully acknowledged.

\section{References}

[1] N. Dovichi, J. Zhang, Angew. Chem., Int. Ed. Engl. 39 (2000) 4463.

[2] N.A. Polson, M.A. Hayes, Anal. Chem. 73 (2001) 312.

[3] A. Nagaki, M. Togai, S. Suga, N. Aoki, K. Mae, J. Yoshida, J. Am. Chem. Soc. 127 (2005) 1166.

[4] R.D. Oleschuk, D.J. Harrison, Trends Anal. Chem. 19 (2000) 379.

[5] K.B. Mogensen, H. Klank, J.P. Kutter, Electrophoresis 25 (2004) 3498.

[6] M. Foquet, J. Korlach, W.R. Zipfel, W.W. Webb, H.G. Craighead, Anal. Chem. 76 (2004) 1618.

[7] J.P. Shelby, D.T. Chiu, Anal. Chem. 75 (2003) 1387.

[8] T. Vilkner, D. Janasek, A. Manz, Anal. Chem. 76 (2004) 3373.

[9] J. Landers (Editor), Handbook of Capillary Electrophoresis, 2nd ed., CRC Press, New York, USA, 1997.

[10] H. Poppe, Anal. Chim. Acta 114 (1980) 59.

[11] J.A. Taylor, E.S. Yeung, J. Chromatogr. 550 (1991) 831.

[12] J.P. Chervet, R.E.J. Vansoest, M. Ursem, J. Chromatogr. 543 (1991) 439.

[13] T.S. Wang, J.H. Aiken, C.W. Huie, R.A. Hartwick, Anal. Chem. 63 (1991) 1372.

[14] N.J. Petersen, K.B. Mogensen, J.P. Kutter, Electrophoresis 23 (2002) 3528.

[15] Y.W. Lin, C.C. Huang, H.T. Chang, Anal. Bioanal. Chem. 376 (2003) 379.

[16] W.L. Tseng, Y.W. Lin, K.C. Chen, H.T. Chang, Electrophoresis 23 (2002) 2477.

[17] H. Salimi-Moosavi, Y.T. Jiang, L. Lester, G. McKinnon, D.J. Harrison, Electrophoresis 21 (2000) 1291.

[18] M.L. Adams, M. Enzelberger, S. Quake, A. Scherer, Sens. Actuators, A 104 (2003) 25.

[19] A. O'Keefe, D. Deacon, Rev. Sci. Instrum. 59 (1988) 2544.

[20] J.J. Scherer, J.B.P. O’Keefe, R.J. Saykally, Chem. Rev. 97 (1997) 25.

[21] M.D. Wheeler, S.M. Newman, A.J. Orr Ewing, M.N.R. Ashfold, J. Chem. Soc., Faraday Trans. 94 (1998) 337.

[22] S.S. Brown, Chem. Rev. 103 (2003) 5219.

[23] G. Berden, R. Peeters, G. Meijer, Int. Rev. Phys. Chem. 19 (2000) 565 . 
[24] M. Jakubinek, Z. Tong, S. Manzhos, H.-P. Loock, Can. J. Chem. 82 (2004) 873.

[25] G. Meijer, M.G.H. Boogaarts, R.T. Jongma, D.H. Parker, A.M. Wodtke, Chem. Phys. Lett. 217 (1994) 112.

[26] D. Romanini, A.A. Kachanov, N. Sadeghi, F. Stoeckel, Chem. Phys. Lett. 264 (1997) 316.

[27] B.A. Paldus, A.A. Kachanov, Can. J. Phys. 83 (2005) 975.

[28] B. Bahnev, L. van der Sneppen, A.E. Wiskerke, F. Ariese, C. Gooijer, W. Ubachs, Anal. Chem. 77 (2005) 1188.

[29] H. Xu, N. Shafer-Ray, F. Merkt, D.J. Hughes, M. Springer, R.P. Tuckett, R.N. Zare, J. Chem. Phys. 103 (1995) 5157.

[30] K.L. Bechtel, R.N. Zare, A.A. Kachanov, S.S. Sanders, B.A. Paldus, Anal. Chem. 77 (2005) 1177.

[31] D. Kleine, J. Lauterbach, K. Kleinermanns, P. Hering, Appl. Phys. B: Lasers Opt. 72 (2001) 249.

[32] R. Engeln, G. von Helden, A.J.A. van Roij, G. Meijer, J. Chem. Phys. 110 (1999) 2732.

[33] S.L. Logunov, Appl. Opt. 40 (2001) 1570.

[34] R.N. Muir, A.J. Alexander, Phys. Chem. Chem. Phys. 5 (2003) 1279 .

[35] A.C.R. Pipino, J.W. Hudgens, R.E. Huie, Rev. Sci. Instrum. 68 (1997) 2978.

[36] A.C.R. Pipino, J.W. Hudgens, R.E. Huie, Chem. Phys. Lett. 280 (1997) 104

[37] A.C.R. Pipino, Phys. Rev. Lett. 83 (1999) 3093.

[38] A.C.R. Pipino, J.P.M. Hoefnagels, N. Watanabe, J. Chem. Phys. 120 (2004) 2879.

[39] T. von Lerber, M.W. Sigrist, Appl. Opt. 41 (2002) 3567.
[40] D.E. Vogler, M.G. Muller, M.W. Sigrist, Appl. Opt. 42 (2003) 5413.

[41] M. Gupta, H. Jiao, A. O'Keefe, Opt. Lett. 27 (2002) 1878.

[42] G. Stewart, K. Atherton, H.B. Yu, B. Culshaw, Meas. Sci. Technol. $12(2001) 843$.

[43] G. Stewart, P. Shields, B. Culshaw, Meas. Sci. Technol. 15 (2004) 1621.

[44] N. Trefiak, J.A. Barnes, F. Rask, D.G. Courtney, R. Walford, R. Li, R.D. Oleschuk, H.-P. Loock, Proc. SPIE 5969, 596900 (2005) (DOI: doi:10.1117/12.629756).

[45] P.B. Tarsa, P. Rabinowitz, K.K. Lehmann, Chem. Phys. Lett. 383 (2004) 297.

[46] C.J. Wang, S.T. Scherrer, Appl. Opt. 43 (2004) 6458.

[47] C. Vallance, New J. Chem. 29 (2005) 867.

[48] R. Engeln, G. von Helden, G. Berden, G. Meijer, Chem. Phys. Lett. 262 (1996) 105.

[49] Z. Tong, A. Wright, T. McCormick, H.-P. Loock, Anal. Chem. 76 (2004) 6594.

Hans-Peter Loock is an Associate Professor in the Department of Chemistry at Queen's University, Canada. He received a B.Sc. degree in Engineering Chemistry in 1991 (Technical University Darmstadt, Germany) and a Ph.D. degree in 1996 from the University of Victoria, Canada. After two years postdoctoral work at the National Research Council of Canada, he joined Queen's University. His research interests are in laser spectroscopy, photodissociation dynamics and molecular physics, but have more recently expanded to include the application of laser spectroscopic techniques to analytical devices. 